\title{
Multitrait-multiparity model for joint genetic evaluation of Nordic bulls for udder health traits
}

\author{
Enyew Negussie, Minna Koivula and Esa Mäntysaari \\ Agrifood Research Finland, Animal Production Research, Animal Breeding, Jokioinen, Finland. \\ Correspondence to: enyew.negussie@mtt.fi
}

\begin{abstract}
Introduction
Somatic cell count and clinical mastitis are traits that are directly related to udder health. Because of their direct influence on costs of milk production they are generally considered to be an important factor in maximizing profit from dairy industry globally. Nordic countries (Denmark, Finland, Norway and Sweden) are the only countries with well-established, national recording systems for health data in dairy cattle and the only to include clinical mastitis (CM) directly in the breeding programs. Additionally, the longstanding exchange of genetic material (bull semen) across Nordic countries has created a sufficient genetic link between dairy populations which could be utilized for improving udder health through joint across-country testing and evaluation of bulls and cows. Joint testing and evaluation between Nordic countries allows accurate comparison and selection of bulls and cows across borders and leads eventually to more efficient utilization of knowledge and resources in genetic evaluation (Juga, 2002). There are, however, some differences between countries in terms of the definition of udder health traits, the parameters, number of lactations considered and most importantly the models used for the genetic evaluation. In most cases the models used so far consider SCC and CM separately in genetic evaluation of dairy bulls and cows. Basically the inclusion of traits genetically correlated to mastitis in the same evaluation can enhance breeding for increased resistance to mastitis. Besides, the statistical information contained in a categorical trait like CM is small and therefore a multi-trait model i.e. a combined analysis of mastitis with a correlated trait (SCC) as an additional source of information will be preferred to increase the accuracy of genetic evaluation. The main objective of this study was to develop multitrait-multiparity (MT-MP) model for the joint genetic evaluation of Nordic bulls for udder health traits.
\end{abstract}

\section{Material and methods}

Data

The data used was obtained from 1.1 million Finnish and 1.0 million Swedish Ayrshire cows. Lactations 1-3 were considered and lactation average SCC and CM observations were combined by lactation for each cow prior to evaluation. As a result, the Finnish, Swedish and the joint data set contained 2.28, 2.21 and 4.49 million combined (SCC+CM) records. Although there are slight differences in the definition of traits between the two countries, traits used in this study were defined as follows: SCC was defined as lactation mean of $\log$ transformed $\left(\log _{\mathrm{e}} \mathrm{SCC}\right)$ test day somatic cell count in $(1000 \mathrm{cells} / \mathrm{ml})$ and $\mathbf{C M}$ was defined as " 0 " or " 1 " depending on whether or not a cow had at least one veterinary treatment for mastitis within the period from 7 days before to 150 days after calving. Culling due to udder diseases with in the above period was also used as additional information. The Ayrshire pedigree used was compiled based on information obtained form Finland, Sweden and interbull sire cross-reference files. The pedigrees of 10,381 bulls with daughters were traced through sires and maternal grand sires and in total the joint pedigree contained about 11,281 bulls.

\section{Models and genetic parameters}

SCC and CM in the first three lactations were considered as different traits. The necessary genetic parameters for the estimation of breeding values were obtained from variance co-variance components estimated based on sample of data extracted from the joint data set by DMU package using AI REML. A linear bivariate sire model was used for the estimation of the parameters and results obtained are presented in Table 1. 
Table 1. Genetic parameters heritability (on the diagonal) and genetic correlations (below diagonal) used in the Ayrshire test-runs (SCC1, SCC2, SCC3, CM1, CM2, and CM3 stands for SCC and CM in the $1^{\text {st }}$, $2^{\text {nd }}$ and $3^{\text {rd }}$ lactations, respectively).

\begin{tabular}{lllllll}
\hline Traits & SCC1 & SCC2 & SCC3 & CM1 & CM2 & CM3 \\
\hline SCC1 & $\mathbf{0 . 1 3 7}$ & & & & & \\
SCC2 & 0.899 & $\mathbf{0 . 1 2 2}$ & & & & \\
SCC3 & 0.811 & 0.981 & $\mathbf{0 . 1 1 9}$ & & & \\
CM1 & 0.721 & 0.664 & 0.577 & $\mathbf{0 . 0 2 3}$ & & \\
CM2 & 0.654 & 0.704 & 0.681 & 0.868 & $\mathbf{0 . 0 2 0}$ & \\
CM3 & 0.577 & 0.696 & 0.687 & 0.759 & 0.955 & $\mathbf{0 . 0 2 2}$ \\
\hline
\end{tabular}

Models

Since November 2003 in Finland genetic evaluation for SCC is calculated using a random regression testday model (Negussie et al. 2003) while CM is evaluated using single trait repeatability sire model. On the other hand, in Sweden sire model was used for genetic evaluation of both SCC and CM considering only first lactation records. Although there are some differences between countries in the type of models used for the national genetic evaluation of SCC and CM the effects included in the models are basically similar. Therefore, based on both national models the following MT-MP sire model was tested for the joint genetic evaluation of Finnish and Swedish bulls for udder health traits. The MT-MP model used for Ayrshire data included fixed effects of age*parity*country, herd-5year period (HY5), and YM*country, and random effects of Herd-1year period (HY), sire and residual effects. The mathematical representation of the complete bivariate model involves the first three lactations of SCC and CM. The model for the three lactation of a trait was as follows:

$$
\mathbf{Y}_{i}=\left[\begin{array}{lll}
\mathbf{X}_{\mathrm{i} 1} & & \\
& \mathbf{X}_{\mathrm{i} 2} & \\
& & \mathbf{X}_{\mathrm{i} 3}
\end{array}\right]\left[\begin{array}{l}
\mathbf{b}_{\mathrm{i} 1} \\
\mathbf{b}_{\mathrm{i} 2} \\
\mathbf{b}_{\mathrm{i} 3}
\end{array}\right]+\left[\begin{array}{lll}
\mathbf{w}_{\mathrm{i} 1} & & \\
& \mathbf{W}_{\mathrm{i} 2} & \\
& & \mathbf{w}_{\mathrm{i} 3}
\end{array}\right]\left[\begin{array}{l}
\mathbf{h y}_{\mathrm{i} 1} \\
\mathbf{h y}_{\mathrm{i} 2} \\
\mathbf{h y}_{\mathrm{i} 3}
\end{array}\right]+\left[\begin{array}{lll}
\mathbf{z}_{\mathrm{i} 1} & & \\
& \mathbf{z}_{\mathrm{i} 2} & \\
& & \mathbf{z}_{\mathrm{i} 3}
\end{array}\right]\left[\begin{array}{l}
\mathbf{s}_{\mathrm{i} 1} \\
\mathbf{s}_{\mathrm{i} 2} \\
\mathbf{s}_{\mathrm{i} 3}
\end{array}\right]+\mathbf{e}_{i}
$$

where $\mathbf{Y}_{\mathrm{i}}$ is a vector of records ( $\mathrm{i}=\mathrm{SCC}$ or CM); $\mathbf{b}_{\mathrm{i}}$ is a vector of fixed effects, $\mathbf{h} \mathbf{y}_{\mathrm{i}}$ is a vector of random herd-year effects, $\mathbf{s}_{\mathrm{i}}$ is a vector of breeding values and $\mathbf{e}_{\mathrm{i}}$ is a vector of residual effects and $\mathbf{X}_{\mathrm{i}}, \mathbf{W}_{\mathrm{i}}$ and $\mathbf{Z}_{\mathrm{i}}$ are incidence matrices that link the effects to $\mathbf{Y}_{\mathrm{i}}$. In the bivariate model we have $\mathbf{Y}=\left[\begin{array}{l}\mathbf{Y}_{1} \\ \mathbf{Y}_{2}\end{array}\right]$ and similarly for other vectors in the model. The effects of herd-year, additive genetic (s) and residual (e) were assumed random with mean zero and $\operatorname{var}(\mathbf{h y})=\mathbf{H} \otimes \mathbf{I}, \operatorname{var}(\mathbf{s})=\mathbf{G}_{0} \otimes \mathbf{A}$, and $\operatorname{var}(\mathbf{e})=\mathbf{R}_{0} \otimes \mathbf{I}$, where $\mathbf{I}$ is an identity matrix and $\mathbf{A}$ is a matrix of additive genetic relationship among animals; $\mathbf{H}, \mathbf{G}_{\mathrm{o}}$ and $\mathbf{R}_{\mathrm{o}}$ are 6 × 6 variance covariance matrices for herd-year, additive genetic and residual effects and $\otimes$ denotes a Kronecker product. Mixed model equations were solved by preconditioned conjugate gradient (PCG) method with iteration on data technique using the MIX99 software (Stranden and Lidauer, 1999).

\section{Results and discussion}

The average SCC and incidence of CM (\%) in Finland and Sweden by lactation number is given in Table 2. It shows that the mean SCC by parity is more variable in the Swedish than in the Finnish Ayrshire data. In general, in both cases SCC in the first parity was lower and tends to increase gradually with the increase in the lactation number. The incidence of $\mathrm{CM}$ also differs over parities and between countries. Similar to the trend in SCC, there is a lower incidence of CM in the $1^{\text {st }}$ lactation cows than in the $2^{\text {nd }}$ and $3^{\text {rd }}$ lactation cows. In Finland, the average incidence of CM over parities is slightly higher than that observed in Sweden. 
Table 2. Phenotypic mean and standard deviation (in brackets) for SCC and incidence of CM (\%) by country and lactation number

\begin{tabular}{|c|c|c|c|c|}
\hline \multirow{2}{*}{ Lactation No. } & \multicolumn{2}{|c|}{ Finland } & \multicolumn{2}{c|}{ Sweden } \\
\cline { 2 - 5 } & SCC & CM & SCC & CM \\
\hline $\boldsymbol{I}$ & $4.28(09)$. & $9.6(0.2)$ & $3.97(0.9)$ & $7.3(0.2)$ \\
\hline $\mathbf{2}$ & $4.30(0.9)$ & $9.9(0.2)$ & $4.14(1.0)$ & $8.8(0.2)$ \\
\hline $\mathbf{3}$ & $4.32(0.9)$ & $11.9(0.3)$ & $4.43(1.1)$ & $11.2(0.3)$ \\
\hline
\end{tabular}

\section{Univariate vs. bivariate model}

The correlation between EBVs from joint bivariate and joint univariate models for SCC and CM were compared in the different groups of Ayrshire bulls (i.e. bulls born from 1991-93 and from 1995-97) with at least 50 daughters. In both cases, the correlation for SCC traits were 0.99 while for CM the estimate ranged form 0.77 to 0.84 with the relatively lower estimates obtained in the second group of bulls (i.e. bulls born from 1995-97). The result in general shows that in moving from univariate to bivariate model there will be little or no change in the estimates of EBVs for SCC in Ayrshire bulls. However, the change in the evaluation of sires for CM was significant. The genetic trend for SCC and CM from joint bivariate and joint univariate models were also assessed and presented in Figures 1 and 2, respectively. As expected from the presented correlations the genetic trends obtained for SCC from the two models were very close to each other. However, the trend observed for CM from bivariate and univariate models were slightly different and the univariate model tends to underestimate the genetic trend. The fact that for SCC there is little or no significant difference between the two models also indicates that it is CM that will benefit more from the covariances between the traits that is used in the evaluation applying the bivariate model. The two models were also contrasted in terms of the magnitude of bias involved in the prediction of breeding values. In this regard the regression of EBVs from the whole recent data containing new information on EBVs obtained from a reduced earlier data showed that the bias in prediction of EBVs is significantly larger in the univariate than in the bivariate model.

\section{Mean and standard deviation of breeding values}

The mean and standard deviation (sd) of EBVs from bivariate and univariate models were compared for Ayrshire bulls born from 1994-95 with at least 50 daughters. The result obtained showed that compared to the univariate model the mean and the sd of breeding values were higher for estimates obtained from the bivariate model. The difference in sd of EBVs revealed that for SCC no significant difference was observed between the two models $(<1.0 \%)$. For CM, however, the difference was significant and the sd of the EBVs from the bivariate model was higher than the univariate model by $9.4,12.5$ and $19.5 \%$ for CM1, CM2 and CM3, respectively. This shows that faster genetic progress will be achieved in reducing the incidences of CM if bulls were selected based on bivariate than univariate model.

\section{Joint vs National model}

The correlation of EBVs and genetic trends for SCC and CM obtained from the joint model (Finnish + Swedish) were compared to the results from the national models. For a general acceptance of a joint model the assumption is that the model should be as good as the national models. In this regard, the correlations obtained between the EBVs from the joint and Finnish model ranged from 0.91 to 0.98 for SCC and 0.94 to 0.97 for CM. Similarly the correlations between the Swedish national and the joint model ranged from 0.98 to 0.99 for SCC and 0.94 to 0.99 for CM. The observed Finnish national genetic trends for SCC and CM were very close to the trend observed from joint model indicating a good agreement between the joint and national models.

\section{Conclusions}

The genetic trend for SCC from bivariate and univariate models were very close and the correlation between them was high (0.99). However, the lower correlation (0.77-0.84) and a slightly different genetic trend for $\mathrm{CM}$ indicates that in moving from univariate to bivariate model more of the changes in EBVs will be in CM than in SCC. The analysis for the possible bias in the prediction of EBV due to the addition 
new information over time showed that the magnitude of bias observed with univariate model is higher than the bivariate model. The comparison of the mean and SD of EBVs of a selected group of bulls indicated that more genetic progress in CM would be realized if selection of bulls for CM is based on bivariate model than on univariate model.

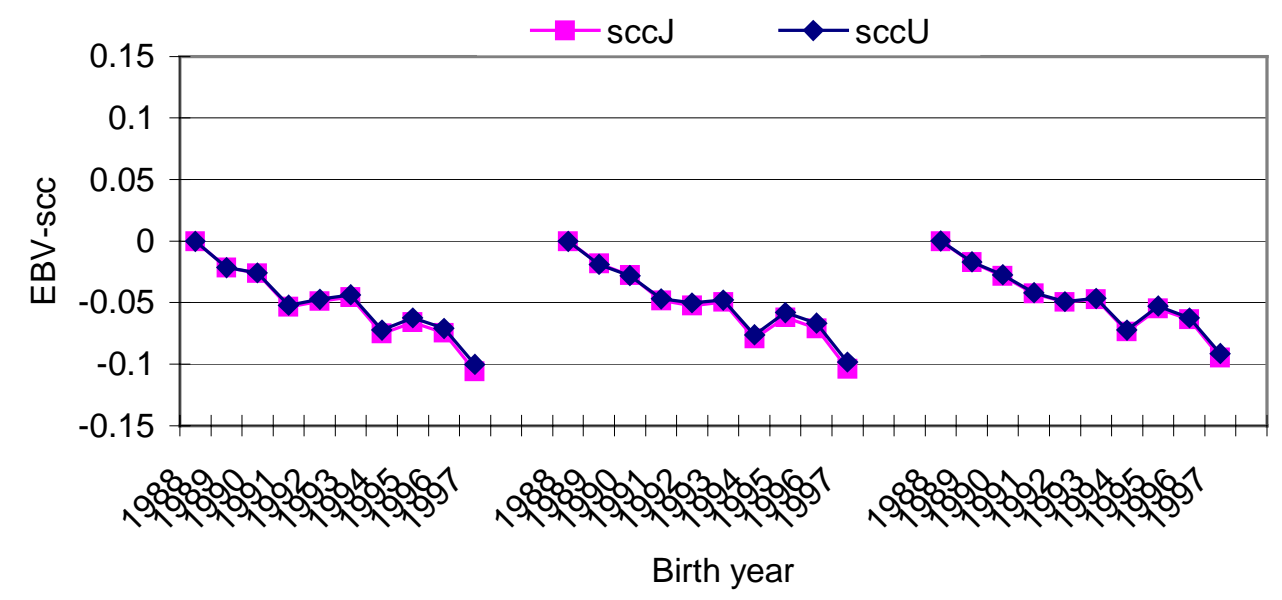

Figure 1. Genetic trend for SCC from joint bivariate model (SCCJ) vs joint univariate model (SCCU)

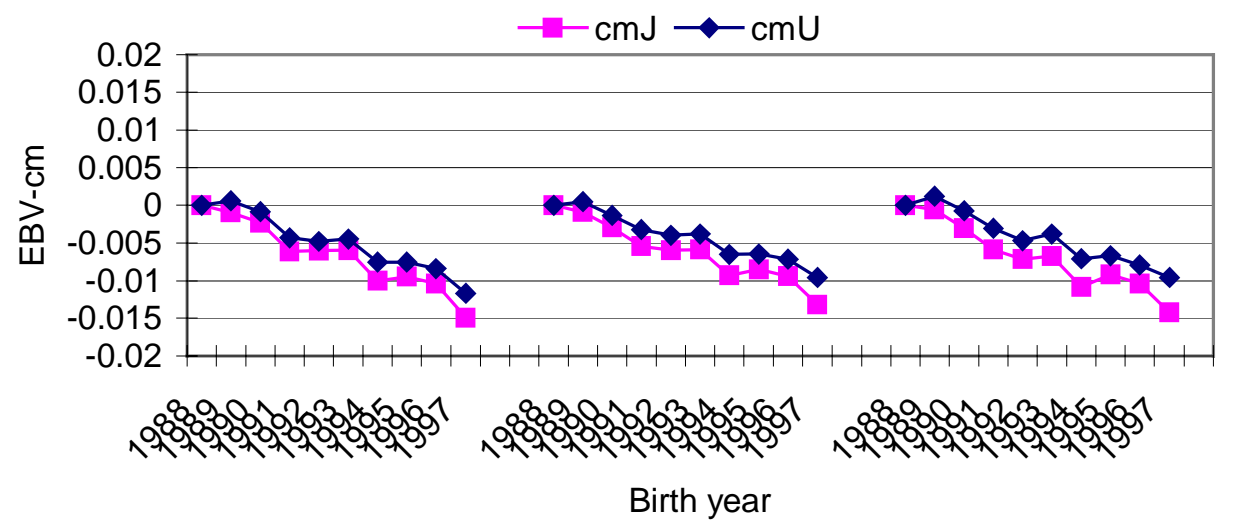

Figure 2. Genetic trend for CM from joint bivariate model $(\mathrm{cmJ})$ vs joint univariate model $(\mathrm{cmU})$

\section{References}

Juga, J. 2002. Joint Nordic evaluation as a first step to joint breeding program. Interbull Bulletin No. 29:133-137 Negussie, E., Koivula, M. and Mäntysaari, E.A. 2003. Multiparity random regression test-day model for genetic evaluation of dairy cattle for somatic cell count (SCC). Livest. Prod. Sci. (Submitted).

Stranden, I., Lidauer, M. 1999. Solving large mixed linear models using preconditioned conjugate gradient iteration. J. Dairy Sci. 82:2779-2787 